\title{
The Reverse-flow Radial Forearm Flap for Soft Tissue Reconstruction of the Wrist and Hand
}

\author{
*Tarek Abulezz , **Ahmed Gaber , *Mohamed Abdelaal, *Sherif Bakri* \\ *Plastic Surgery Department, Sohag University Hospital, Faculty of Medicine, Sohag \\ University, Egypt \\ **Lecturer of Plastic Surgery, Faculty of Medicine, Sohag University , Egypt
}

\begin{abstract}
Soft-tissue injuries of the hand and wrist regions are quiet common casualty. Flap coverage is often required to achieve primary wound closure and to achievea good functional result. The use of the reversed flow radial forearm fasciocutaneous flap in the reconstruction of the hand and wrist soft tissue defectshas been described by many authors to be an easy-to-learn and very versatile technique in hand injuries and reconstruction.In this report, the anatomy and vascular basis of the reversed flow radial forearm flap are presented along with the evaluation of its application in twenty patients with different hand and wrist soft tissue defects.
\end{abstract}

Keywords: radial forearm, reverse-flow flap, hand reconstruction, fasciocutaneous, thumb reconstruction

\section{Introduction}

The hand is the primary organ used by humans to deal with and interact with the external environment. Hand and wrist regions are frequently involved in trauma resulting in soft tissue defects. Due to the paramount functional importance, these injuries should be carefully evaluated to determine the most appropriate method of repair. Careful identification and reposition of anatomical structures are sometimes all what we need in simple injuries provided no tissue or components lost. Split thickness skin grafts may be suitable only for healthy granulating wounds. In spite of being easy, cheap and quick, skin grafts cannot be used where there is an exposed bone, joint, tendons, nerves or blood vessels. However, complex injuries around the wrist usually result in loss of skin and soft tissue with exposure of the tendons and bones. Hand injuries which involve substantial skin and soft tissue loss require immediate soft tissue cover to achieve early wound closure and to minimize wound infection, fibrosis and scarring. This soft tissue reconstruction should not only address the immediate need for wound closure but also the possibility of further surgical intervention like tendon repairs[1]. In these scenarios, the reconstructive hand surgeon have to pursue more advanced and even sophisticated techniques for wound coverage based upon knowledge of the available pedicled and free flaps. Local transposition flaps can be used to reconstruct small defects in one-stage reconstruction procedure; however, they are inadequate for medium-sized and large or complex defects where distant flaps from the abdomen or groin are usually used. The main drawbacks are the significant periods of post-operative immobilization in awkward positions and multi-staged nature of the techniques. Free flaps may be the best option however; they demand prerequisites of availability of technical 
experience and microsurgical equipments. One of the major advantages of free flaps compared to groin or abdominal flaps is the early mobilization of the reconstructed hand[2].

The radial forearm flap was developed as a free fasciocutaneous flap in 1978 by Yang Goufan and coworkers in China[3]. Originally, it was used in reconstruction of head and neck defects after tumor resection or post burn contracture release $[4,5]$. The free radial forearm flap was then used for reconstruction of upper extremity; however, it was soon recognized that the flap could be used as a distally based reverse-flow pedicled flap that can reach and cover the palmar and dorsal surfaces of the wrist and the hand[6,7].

The arterial supply of the flap depends on retrograde flow through the ulnar artery and palmar arches after ligation of the proximal radial artery[8]. The venous drainage of the flap is based upon a "crossover pattern" and a "bypass pattern" between the 2 accompanying venae comitantes, as well as communication between the cephalic vein and deep venous system [9, 10]. Reconstruction of hand injuries have been described and advocated by many authors, thereby avoiding a free flap, as long as there is adequate collateral flow through the ulnar $[1,11]$.

\section{Patients and Methods}

In the period between February 2009 and July 2016, 20 patients underwent reverse radial forearm flap (RRFF) reconstruction for a variety of wrist and hand defects due to trauma or after releasing contractures of old burn injuries at the Department of Plastic Surgery, Sohag University Hospital, Egypt. All patients were assessed preoperatively with the Allen test and color Doppler ultrasonography to confirm the adequacy of the ulnar artery. Each RRFF was harvested in the same technique including forearm fascia, radial artery, cephalic vein and vena comitans. The flaps were elevated with an arm pneumatic tourniquet inflated to $160 \mathrm{mmHg}$ for a maximum of $90 \mathrm{~min}$. The donor site was closed by a splitthickness skin graft in the majority of patients but in 3 patients the secondary defect was closed directly with no need for the skin graft. The dressing was maintained with elastic bandage and the first dressing for the donor site was done in the 6th day postoperative. The flap was having a separate dressing to facilitate monitoring and follow-up.

\section{Results}

Twenty patients 16 male and 4 females were included in this study. Nine flaps were in the left and 11 in the right forearm. The size of the skin paddle of the flap ranged from $5 \times 3$ $\mathrm{cm}$ to $8 \times 8 \mathrm{~cm}$ with an average surface area of $38.4 \mathrm{~cm} 2$. The donor site was closed by a split-thickness skin graft in the majority of patients but in 3patients the secondary defect was closed directly with no need for the skin graft. The dressing was maintained with elastic bandage and the first dressing for the donor site was done in the 6th day postoperative. The flap recipient site was dressed separately to facilitate monitoring and follow-up. In two cases, there was mild congestion in the flaps that resolved within 2 days with elevation of the hand and maintaining the patient on low molecular weight heparin. In one flap, there was a partial flap necrosis of a $5 \mathrm{~mm}$ strip at the distal end of the flap that healed with secondary intension. Four donor sites became hypertrophic and necessitate the use of topical steroid creams together with pressure garment for 3 months. 


\section{Case Presentation}

Patient \# 1:A 24-year-old man presented with crush injury to his right hand, exposed MP joint of index finger, 2nd metacarpal bone, and laceration of extensor tendon of index finger (Fig 1a). After wound debridement and extensor tendon repair, distally based radial forearm flap with a $5-7-\mathrm{cm}$ skin paddle was used to provide durable soft-tissue. The donor site was skin grafted simultaneously. Accepted cosmetic healing occurred (Fig 1b).

\section{Patient \#2:}

Female patient 12 ys old presented with electrical burn injury of right hand with raw area on the volar aspect of thumb, loss flexor pollicus tendon and exposed bone of proximal phalanx (fig2a). Debridement of necrotic tissue and coverage by distally based radial forearm flap was performed, donor site closed by split thickness graft, Accepted cosmetic healing occurred (Fig 2b).

\section{Figures}

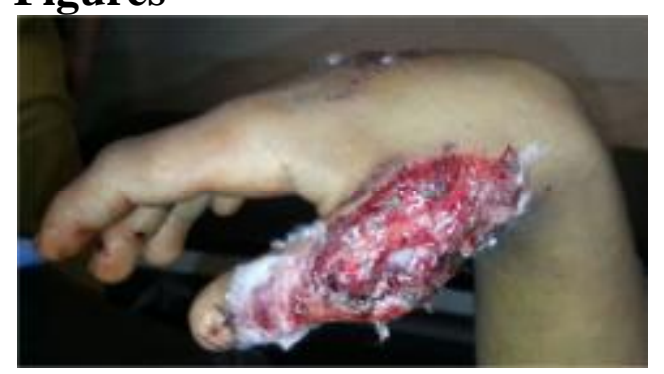

Figure 1a

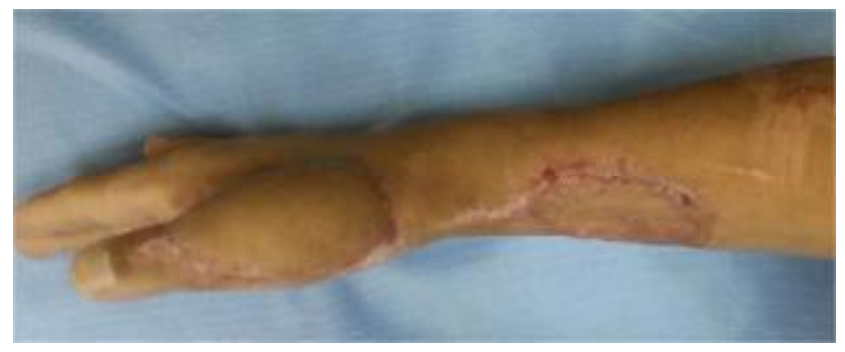

Figure 1b
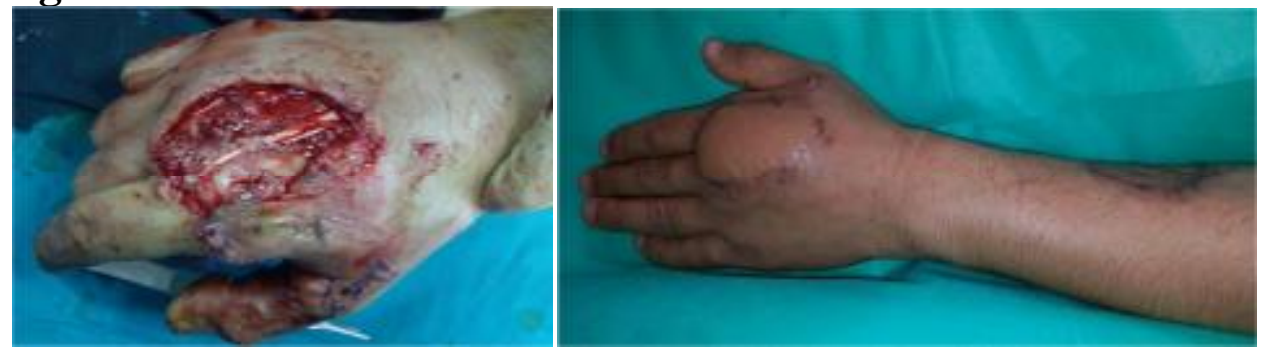

Figure 2a

Figure 2b 


\section{Discussion}

Skin grafts cover will be inappropriate in such situations. Crush and avulsion injuries of the dorsum of wrist and hand are common examples of such difficult soft tissue defects in our environment. In the past, distant flaps from the trunk especially the groin flap 16 had dominated the reconstructive options of such defects, but the groin flap suffers from the same disadvantages of distant flaps in hand reconstruction. It is a multi-staged procedure requiring immobilization of the hand in a dependent position for two to three weeks postoperatively to allow for safe flap division and inset 5 . Edema of hand, interference with hand physiotherapy, stiffness of the ipse-lateral shoulder joint tends to militate against hand function. Its use in hand reconstruction is therefore on the decline. The distally based island radial forearm flap was our preferred option for the case 2 patient. Apart from the ease of elevation of the flap, it was a single staged procedure providing thin and supple skin for coverage of the dorsum of wrist joint and hand up to the finger knuckles. Hand elevation was possible in the immediate postoperative period and physiotherapy exercises were unhindered. Several authors $[1,5,11,12]$ have previously reported on the advantages of distally based island radial forearm flap in hand reconstruction. It can also transfer vascularised bone, tendon and nerve to reconstruct composite defects[5].

Our third patient with traumatic amputation at the mid palmar level had the amputation stump covered with a distally based island radial forearm flap. Again it provided primary wound closure in one stage. In this patient consideration was given for the use of split skin graft cover which is simpler.
However the amputation defect was irregular with bones sticking out. Split skin graft coverage under such situation will require bone shortening which the patient declined. Also considered was the possibility of future reconstruction to improve hand function and a flap cover will provide a more dependable soft tissue.

The radial forearm flap has also been used as fascial rather than fasciocutaneous flap[12-15].Some of the advantages of the fascial flaps are the reduction in the donor site deformity which can be closed directly, avoidance of hair in palmer reconstruction in hirsute individuals and reduction of the thickness of subcutaneous tissue in some patients particularly females[12].In our fourth patient, we required thinner tissue than would have been provided by a fasciocutaneous flap. Split thickness skin graft alone would have led to recurrence of contracture and we reasoned that fascial flap covered with skin graft will provide tissue of adequate thinness with less possibility of contracture recurrence. The direct closure of the donor site in this patient was an added advantage. Use of skin grafted fascial flaps in hand reconstruction has also been previously reported[15].

The major concerns when using the radial forearm fasciocutaneuos flap had been the donor site morbidity[5]. These include the sacrifice of a major source artery to the forearm and hand and the wound healing problems and poor aesthetic appearance of the donor site of the flap which most often requires to be skin grafted. To avoid hand ischaemia when using the radial artery forearm flap, it is important to establish that the rest of the hand will survive on the ulnar artery input alone. A timed Allen test is 
therefore mandatory[1]. Allen test requires that the patient makes a tight fist to exsanguinate the hand. This obviously presents a problem in acute hand trauma. In such situations some authors have proposed intra-operative Allen test by clamping the radial artery either proximally or distally[1, 12]. However in two of our patients with acute hand trauma we rather exsanguinated the hand under general anesthesia and proceeded with Allen test. Although ulnar artery flap is another forearm flap described for both elbow, hand and wrist coverage, it has not been popular with us and others[12] because the ulnar artery is commonly the dominant blood supply to the hand. Posterior interosseous artery flap based on the posterior and anterior interosseous arteries and dorsal carpal arch has also been used for hand coverage[16]. Its major advantage is that it does not sacrifice any of the major source vessels to the forearm and hand. However its dissection is quite delicate and the extent of coverage in the wrist and hand is much less than the radial forearm flaps[12].

The wound healing problems and appearance of donor site of the radial forearm flap are well documented[1, 17, 18]. Complications are more in composite flaps than in ordinary fasciocutaneous flap[18]. The skin grafted donor site usually produces poor aesthetic results. Various authors have suggested ways to minimize this[18, 19]. We agree with others[1] that the handling of the donor site should be quite meticulous and possibly its closure should not be delegated to the inexperienced member of the team. The possibility of scar hypertrophy is usually discussed with the patient since most of our patients are blacks and therefore prone to scar hypertrophy and keloids. However the scar hypertrophy seen in these patients were mainly in the skin grafted donor sites and tended to subside with time.

Conclusions: The technical experiences of the surgeon and the availability of equipments and instrument can also modify the reconstructive decision in each particular situation. The reversed flow radial forearm fasciocutaneous flap is versatile and useful in the reconstruction of the hand and wrist soft tissue defects. The flap offers thin, pliable, hairless skin that can cover skin defects of different hand and wrist's regions. This flap is superior to the groin flap for coverage of medium-sized defects and specifically suitable for covering the dorsal surface of wrist and hand virtue to the similar characteristics. It can be of great value to cover defects resulting from release of contractures of the first web space, and for coverage of thumb stumps.

\section{References}

1.Soutar, D.S. and N.S. Tanner, The radial forearm flap in the management of soft tissue injuries of the hand. $\mathrm{Br} \mathrm{J}$ Plast Surg, 1984. 37(1): p. 18-26.

2.Lassner, F., M. Becker, and N. Pallua, [Therapy of soft tissue trauma in the acute phase of severe hand injuries]. Chirurg, 2001. 72(12): p. 1439-45.

3.Song, R., et al., The forearm flap. Clin Plast Surg, 1982. 9(1): p. 21-6.

4.Soutar, D.S., et al., The radial forearm flap: a versatile method for intra-oral reconstruction. Br J Plast Surg, 1983. 36(1): p. 1-8.

5.Hentz, V.R., et al., The radial forearm flap: a versatile source of composite tissue. Ann Plast Surg, 1987. 19(6): p. 485-98.

6.Megerle, K., M. Sauerbier, and G. Germann, The evolution of the 
pedicled radial forearm flap. Hand $(\mathrm{N}$ Y), 2010. 5(1): p. 37-42.

7.Frey, M., The radial forearm flap as a pedicled flap for resurfacing a scarred nose. Ann Plast Surg, 1994. 32(2): p. 200-4.

8.Muhlbauer, W., E. Herndl, and W. Stock, The forearm flap. Plast Reconstr Surg, 1982. 70(3): p. 336-44.

9.Selber, J.C., et al., Venous drainage of the radial forearm flap: comparison of the deep and superficial systems. Ann Plast Surg, 2011. 66(4): p. 347-50.

10.Lin, S.D., C.S. Lai, and C.C. Chiu, Venous drainage in the reverse forearm flap. Plast Reconstr Surg, 1984. 74(4): p. 508-12.

11.Braun, F.M., et al., Technique and indications of the forearm flap in hand surgery. A report of thirty-three cases. Ann Chir Main, 1985. 4(2): p. 85-97.

12.Jones, N.F., R. Jarrahy, and M.R. Kaufman, Pedicled and free radial forearm flaps for reconstruction of the elbow, wrist, and hand. Plast Reconstr Surg, 2008. 121(3): p. 887-98.
13. Jin, Y.T., et al., Reversed island forearm fascial flap in hand surgery. Ann Plast Surg, 1985. 15(4): p. 340-7.

14. Reyes, F.A. and W.E. Burkhalter, The fascial radial flap. J Hand Surg Am, 1988. 13(3): p. 432-7.

15. Cherup, L.L., et al., The radial forearm skin graft-fascial flap. Plast Reconstr Surg, 1990. 85(6): p. 898-902.

16. Zancolli, E.A. and C. Angrigiani, Posterior interosseous island forearm flap. J Hand Surg Br, 1988. 13(2): p. 130-5.

17. Timmons, M.J., et al., Complications of radial forearm flap donor sites. $\mathrm{Br} \mathbf{J}$ Plast Surg, 1986. 39(2): p. 176-8.

18. Richardson, D., et al., Radial forearm flap donor-site complications and morbidity: a prospective study. Plast Reconstr Surg, 1997. 99(1): p. 109-15.

19. Bashir, M.A., et al., "Z-plasty" modification of ulnar-based fasciocutaneous flap for closure of the radial forearm flap donor defect. Ann Plast Surg, 2010. 64(1): p. 22-3. 